\title{
Association between mean platelet volume levels and inflammation in SLE patients presented with arthritis
}

\author{
Sahin Safak ${ }^{1}$, Ali Ugur Uslu ${ }^{2}$, Korkmaz Serdal ${ }^{2}$, Tasliyurt Turker ${ }^{1}$, Senel Soner ${ }^{3}$, Akyol Lutfi ${ }^{4}$
}

\begin{abstract}
1. Gaziosmanpasa University, Faculty of Medicine, Department of Internal Medicine, Tokat, Turkey
2. Cumhuriyet University, Faculty of Medicine, Department of Internal Medicine, Sivas, Turkey

3. Erciyes University, Faculty of Medicine, Department of Rheumatology, Kayseri, Turkey

4.Bozok University, Faculty of Medicine, Department of Internal Medicine, Yozgat, Turkey
\end{abstract}

\begin{abstract}
Background: Systemic lupus erythematosus (SLE) may be characterized by periods of remissions and chronic or acute relapses. The complexity of clinical presentation of the SLE patients leads to incorrect evaluation of disease activity. Mean platelet volume (MPV) has been studied as a simple inflammatory marker in several diseases. There is no study in the literature about MPV levels in adult SLE patients with arthritis.

Objectives: We aimed to investigate the MPV levels in the SLE population with arthritis during and between activations.

Methods: The study consisted of 44 SLE patients with arthritis in activation period (Group 1), the same 44 SLE patients with arthritis in remission period (Group 2) and 44 healthy controls (Group 3). Erythrocyte sedimentation rate (ESR), creactive protein (CRP), white blood cell count, platelet count, and mean platelet volume (MPV) levels were retrospectively recorded from patient files.

Results: The mean ages of the SLE subjects were $42 \pm 16$ years, while the mean ages of controls was $41 \pm 17$ years. MPV was significantly lower in Group 1(7.66 $\pm 0.89 \mathrm{fL})$ than in Group $2(8.61 \pm 1.06 \mathrm{fL})$ and Group 3(8.62 $\pm 1.11 \mathrm{fL})(\mathrm{p}<0.0001)$. The differences between groups reached statistical significance.

Conclusions: We suggest that MPV levels decrease in patients with arthritis of SLE activation when compared to the same patients in remission and healthy controls.
\end{abstract}

Key words: Systemic lupus erythematosus, Arthritis, Mean platelet volume

DOI: http://dx.doi.org/10.4314/ahs.v14i4.21

\section{Introduction}

Systemic lupus erythematosus (SLE) is a chronic inflammatory disease of unknown cause that may affect any organ of the body. Arthritis lupus is typically nonerosive, non-deforming, and frequently appears before other manifestations of SLE. It affects predominantly women, especially in their 20 s and 30 s. The clinical course of SLE is characterized by periods of remissions and relapses ${ }^{1}$. The correct evaluation of disease activity is of great importance because of the the complexity of the disease. Common methods including erythrocyte sedimentation rate (ESR) and C-reactive protein (CRP) are not completely in accordance with evaluating activation of SLE with arthritis ${ }^{2,3}$. Anti-double

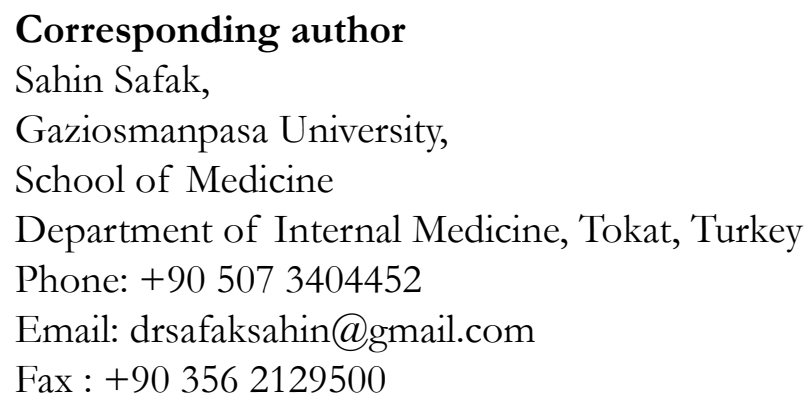

stranded DNA antibody and complement are used to certain clinical manifestations of the disease, especially nephritis, rather than the activity of the disease itself and arthritis ${ }^{4}$. Currently, disease activity in SLE can be assessed using composite disease activity indices, such as the SLE Disease Activity Index (SLEDAI) and British Isles Lupus Assessment Group (BILAG) 5. However, they could be complex for use in routine clinical practice. Therefore, there is a great amount of interest in the identification of biomarkers that can quantify disease activity, although a single biomarker is unlikely to replace clinical evaluation because of the heterogeneity of $\mathrm{SLE}^{6}$.

Mean platelet volume (MPV) is a parameter detected during routine blood count and to which clinicians do not usually pay much attention. Platelet volume is known to be a marker determined from megakaryocytes during platelet production, which is associated with platelet function and activation. Under normal circumstances, there is an inverse relationship between platelet size and number ${ }^{7,8}$. MPV has been studied as a simple inflammatory marker in several diseases. Some studies have reported that MPV increases 
in myocardial infarction and cerebrovascular disease; one hour after the sampling. The complete blood while in contrast, it decreases in active rheumatologic count have been performed in the same analyzer, diseases including rheumatoid arthritis (RA), ankylosing Mindray BC-6800, which is routinely checked every spondylitis and ulcerative colitis ${ }^{9-12}$. To our knowledge, month in the central laboratory of our institution. there is not a study in the literature about MPV levels in adult SLE patients with arthritis. The present Statistical analyses

study aimed to investigate the MPV levels in an adult Data were evaluated using the Statistical Package for SLE population.

\section{Patients and Method} Social Sciences 12.0 program for windows and by

analyzing descriptive statistics (means and standard deviation), comparing the means of quantitative dat The data used in the present study were obtained retro- for more than two groups with Kruskal-Wallis test an spectively from patients who had SLE diagnosis with by comparing dual groups using the Student's t-test. P arthritis complaints based on ACR criteria in August value of 0.05 was considered significant. Correla2011-December 2012 period in Rheumatology Clinic tions between parameters were computed through of the School of Medicine of the Cumhuriyet Univer- Pearson's correlation analysis. Variables found to be sity (Sivas, Turkey). Ethical approval for the study was statistically significant in univariate analyses were obtained from the local ethics committee. The study entered into multivariate logistic regression analysis. consisted of 44 SLE patients with arthritis in activation Multivariate logistic regression models were created period (Group 1), the same 44 SLE patients with arthri- to identify independent predictors of activation of tis in remission period (Group 2) and 44 healthy con- arthritis in SLE patients.

trols (Group 3). The age of SLE patients and healthy controls ranged in 18 to 79 years. Participants with a Results

history of smoking, acute or chronic infectious diseas- Demographic and clinical features of SLE patients es, hemoglobin $>16.5 \mathrm{~g} / \mathrm{dl}$, thrombocytopenia, anemia, are given in Table I.

hypertension, angina pectoris, myocardial infarction, di- The mean ages of the SLE subjects were $42 \pm 16$ years, abetes mellitus, anti-phospholipid syndromes, recurrent while the mean ages of controls was $41 \pm 17$ years. miscarriage, amyloidosis, thrombosis or chronic renal Thirty-three SLE patients (78.6\%) and 35 subjects insufficiency were excluded from the study. ESR, CRP, $(79.5 \%)$ in control group were female. There were no White Blood Cell (WBC) count, platelet count, and significant differences between patient group with SLE MPV levels were retrospectively recorded from patient and healthy controls in terms of age and gender distrifiles. All blood samples were studied within less than bution ( $\mathrm{p}=0.628, \mathrm{p}=0.912$, respectively).

Table I. Main clinical characteristics of the patients with systemic lupus erythematosus and of the controls*

\begin{tabular}{lcc}
\hline & $\begin{array}{c}\text { SLE patients } \\
\mathrm{n}=44\end{array}$ & $\begin{array}{c}\text { Controls } \\
\mathrm{n}=44\end{array}$ \\
\hline Sex, no. female/male & $33 / 11$ & $35 / 9$ \\
Median age, years (range) & $42 \pm 16$ & $41 \pm 17$ \\
Age at SLE onset, years(range) & $32 \pm 8.9$ & - \\
Remission duration, days (range) & $12 \pm 4.9$ & - \\
Butterfly rash & $28 / 44$ & - \\
Discoid lesions & $11 / 44$ & - \\
Photosensitivity & $23 / 44$ & - \\
Mouth ulcers & $14 / 44$ & - \\
Arthralgias/arthritis & $44 / 44$ & - \\
Serositis & $4 / 44$ & - \\
Pleurisy & $4 / 44$ & - \\
Pericarditis & $1 / 44$ & - \\
Renal disease & $12 / 44$ & - \\
Neuropsychiatric manifestations & $4 / 44$ & - \\
Antinuclear antibodies & $41 / 44$ & - \\
Anti-double-stranded DNA & $24 / 44$ & - \\
\hline
\end{tabular}

*Except where indicated otherwise, values are the number
MPV was significantly lower in Group $1(7.66 \pm 0.89 \mathrm{fL}) \quad$ The mean platelet volume and WBC count did not differ than in Grouand Group 3(8.62 $\pm 1.11 \mathrm{fL}) \quad(\mathrm{p}<0.0001)$; among the groups. CRP and ESR values were signifihowever, there was no difference between group 2 cantly higher in Group 1 than Group $2(p<0.0001)$. and group $3(\mathrm{p}=0.772)$. MPV levels were not corre- All study parameters are presented in Table II and III. lated with ESR and CRP.

Table II. Relationship between blood parameters in Group 1and Group 2

\begin{tabular}{lccc}
\hline & $\begin{array}{c}\text { Group } \\
1 \mathrm{n}=\end{array}$ & $\begin{array}{c}\text { Group } \\
2\end{array}$ & $\mathrm{P}$ \\
& 44 & $\mathrm{n}=44$ & \\
\hline Platelet $\left(\mathrm{x} 10^{9} / \mathrm{L}\right)$ & $296.5 \pm 13.5$ & $290.7 \pm 12.1$ & 0.835 \\
$\mathrm{MPV}(\mathrm{fL})$ & $7.66 \pm 0.89$ & $8.61 \pm 1.06$ & 0.0001 \\
$\mathrm{Hb}(\mathrm{g} / \mathrm{dL})$ & $11.9 \pm 1.4$ & $12.3 \pm 1.6$ & 0.356 \\
$\mathrm{Hct}$ & $36.35 \pm 3.70$ & $36.96 \pm 4.69$ & 0.503 \\
$\mathrm{WBC}\left(\mathrm{x} 10^{9} / \mathrm{L}\right)$ & $7.72 \pm 4$ & $8.29 \pm 3.26$ & 0.493 \\
$\mathrm{CRP}(\mathrm{mg} / \mathrm{L})$ & $71.4 \pm 22.3$ & $5.1 \pm 6.1$ & 0.0001 \\
$\mathrm{ESR}(\mathrm{mm} / \mathrm{h})$ & $50.6 \pm 26.9$ & $20.6 \pm 13.9$ & 0.0001 \\
\hline
\end{tabular}

Table III. Relationship between blood parameters in Group 1 and Group 3

\begin{tabular}{lccc}
\hline & $\begin{array}{c}\text { Group } \\
1 \mathrm{n}= \\
44\end{array}$ & $\begin{array}{c}\text { Group } \\
3 \\
\mathrm{n}=44\end{array}$ & $\mathrm{P}$ \\
& $296.5 \pm 13.5$ & $275.5 \pm 45.8$ & 0.333 \\
Platelet $\left(\mathrm{x} 10^{9} / \mathrm{L}\right)$ & & & \\
MPV (fL) & $7.66 \pm 0.89$ & $8.62 \pm 1.11$ & 0.0001 \\
$\mathrm{Hb}(\mathrm{g} / \mathrm{dL})$ & $11.9 \pm 1.4$ & $12.2 \pm 1.7$ & 0.563 \\
Hct & $36.35+3.70$ & $36.93+1.79$ & 0.786 \\
WBC $\left(\mathrm{x} 10^{9} / \mathrm{L}\right)$ & $7.72 \pm 4$ & $7.68 \pm 1.80$ & 0.931 \\
\hline
\end{tabular}


Correlation analysis showed that there was positive cor- $0.556, \mathrm{p}=0.001)$ and negative correlation between MPV relation between CRP $(\mathrm{r}=0.534, \mathrm{p}=0.001)$ and $\mathrm{ESR}(\mathrm{r}=(\mathrm{r}=-0.445, \mathrm{p}=0.001)$ in activation period SLE patients with arthritis (Figure I).

Figure I: Roc-curve analysis of MPV

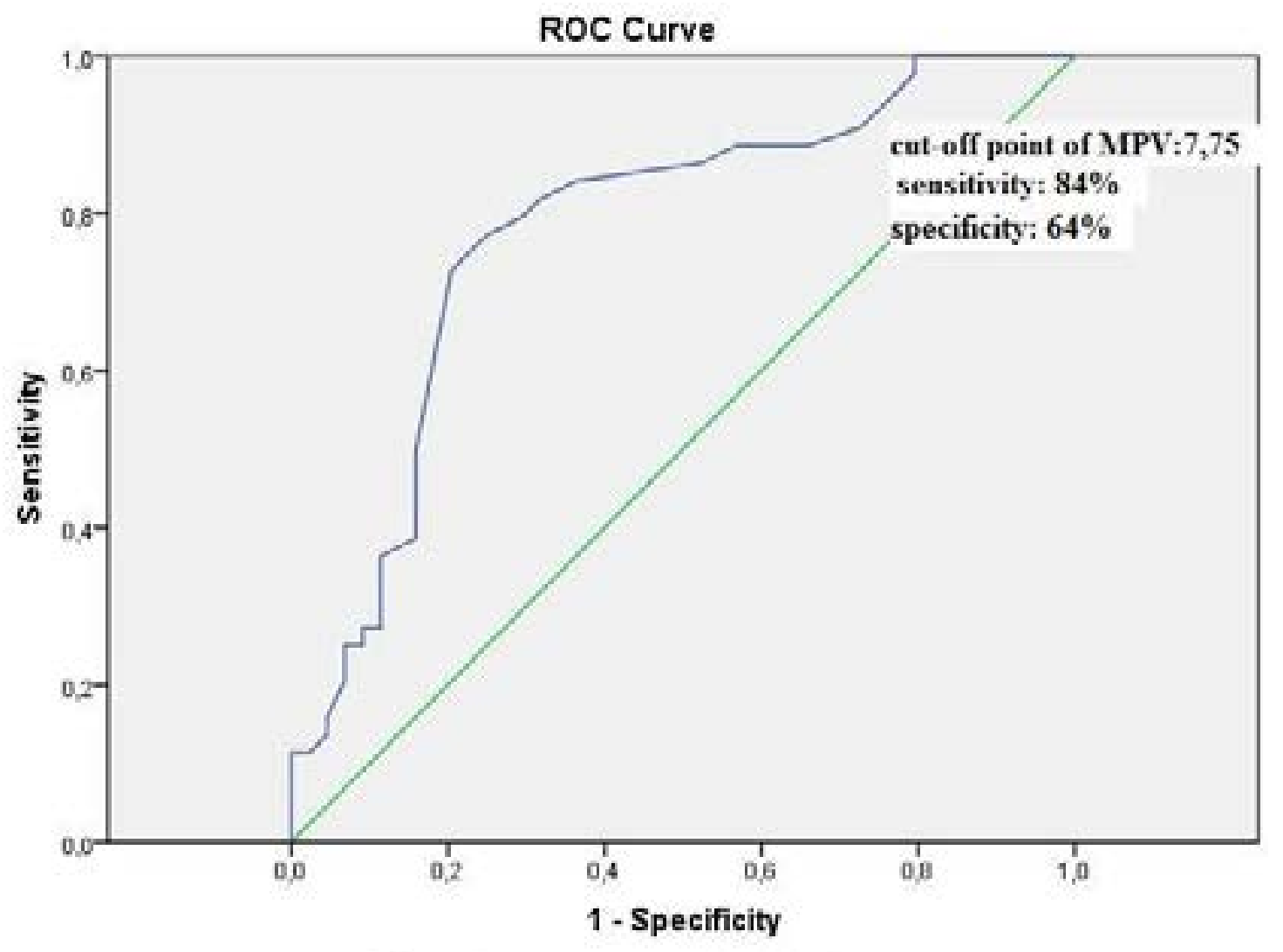

Diagonal segments are produced by ties.

ESR, CRP, MPV, platelet count, and WBC were entered Multivariate analysis showed that only MPV (odds in univariate analysis. CRP, ESR, and MPV were statisti- ratio: $0,179, \% \mathrm{CI}: 0.048-0.671, \mathrm{p}=0.011$ ) and ESR cally important. These values were entered multivariate (odds ratio: $1.134, \% \mathrm{CI}: 1.040-1.235, \mathrm{p}=0.004$ ) were analyses to identify independent predictors of acti- independent predictors of arthritis activation in SLE vation of arthritis in SLE patients. patients (Table IV).

Table IV. Univariate and multivariate analyses

\begin{tabular}{lcccccc} 
& \multicolumn{3}{c}{ Univariate analysis } & \multicolumn{3}{c}{ Multivariate analysis } \\
& $\mathrm{p}$ & Odds ratio & $\mathrm{CI}(\%)$ & $\mathrm{p}$ & Odds ratio & $\mathrm{CI}(\%)$ \\
\hline MPV & 0.001 & 0.327 & $0.181-0.592$ & 0.012 & 0.179 & $0.048-0.671$ \\
ESR & 0.001 & 1.074 & $1.035-1.115$ & 0.004 & 1.134 & $1.040-1.235$ \\
CRP & 0.002 & 1.201 & $1.071-1.347$ & 0.148 & 1.098 & $0.967-1.246$ \\
Platelet count & 0.860 & 1.000 & $1.000-1.124$ & & & \\
WBC & 0.489 & 1.000 & $1.000-1.214$ & & & \\
\hline
\end{tabular}

MPV: Mean platelet volume, WBC: White blood cell, CRP: C-reactive protein, ESR: Erythrocyte sedimentation rate
Two studies have suggested that SLE patients have inIn the present study, we demonstrated that MPV decreased in SLE patients during activation compared with remission and healthy controls. Low levels of MPV were associated with disease activity. We suggest its usefulness as a biomarker for global disease activity and clinical features. In patients with SLE, elevated ESR were found to be associated with disease activity ${ }^{13}$, whereas serum CRP was only slightly elevated and was not correlated with disease activity ${ }^{3,14}$. However, CRP and ESR may present some discordance in reflecting inflammation ${ }^{4}$. Recently, MPV has been considered a reliable marker for indicating platelet activation and inflammation. MPV is easily measured by automated cell counters under any storage conditions for blood samples. MPV is a marker of platelet function and activation, and it is also influenced by inflammation ${ }^{15}$

To our knowledge, there is only one study in the literature about MPV levels in adult SLE patients. In this study, low MPV $(<7.2 \mathrm{fl})$ was recorded by 2 different hematology analyzers in $5(14.3 \%)$ and $23(65.7 \%)$ patients with SLE, mostly at active stage of the disease $(n=28,80 \%) \cdot{ }^{16}$ Results of this study, in which hematological parameters of SLE patients were studied, were in accordance with our results. A major difference of the present study from the ones in literature was that this is the first study evaluating MPV in SLE patients with arthritis in activation and remission periods.

Gasparyan et al. ${ }^{15}$ stated that high-grade inflammation accompanies a decrease of MPV in RA and SLE, possibly due to the increased consumption of large platelets at the sites of rheumatoid inflammation.

Kisacik et al. ${ }^{7}$ found that MPV was low in active ankylosing spondylitis and RA, and that MPV levels increased and then normalized with treatment. In another study lower MPV value of RA patients compared to controls were shown to increase after TNF- $\alpha$ treatment ${ }^{17}$. In the present study, lower MPV values of SLE patients with arthritis were normalized after treatment There are two studies showing that MPV levels of FMF patients during the attack were significantly lower than those of healthy controls ${ }^{18,19}$. MPV has been reported to decrease in some inflammatory bowel diseases such as ulcerative colitis and it could be used for determination of disease activity ${ }^{20}$. This condition is thought to be related to the release of bioactive molecules of pro-inflammatory platelets in the presence of inflammation.

The authors report no conflict of interest.

African Health Sciences Vol 14 Issue 4, December 2014
(1, 22. Of these studies, Naga-

ama et a ${ }^{22}$ showed that plasma platelet-derived micro-

particle levels were significantly higher in SLE patients than in healthy subjects. Furthermore, Nagahama et al stated ${ }^{22}$ that MPV levels were decreased in patients with arthritis of SLE activation as in our study. Several mechanisms for how complement components interact with platelets have been proposed, and most of these theories involve platelet activation.

Common trigger of platelets include immune complexes, which are frequently seen in SLE patients, shear stress due to atherosclerosis or inflammatory cytokines including interferon-alpha ${ }^{21,23,24}$

\section{Limitations}

The results of this study are subjected to some limitations. First, this study was not based on longitudinal observations but was conducted with a retrospective design. Second, since the study is retrospective, clinical activation indices of SLE patients with arthritis (SLEDAI, BILAG) were not calculated. Third, it is a single center study with a relatively small sample size, which might underestimate or overestimate the relationship between MPV and inflammation in SLE patients presented with arthritis. Fourth, the fact that MPV values may be affected by many factors.

\section{Conclusion}

we suggest that MPV levels decrease in patients with arthritis of SLE activation when compared to same patients with remission and healthy controls. Decreased MPV levels were observed in patients with SLE and these results may be related to active inflammatory states. Namely, MPV is associated with disease activity. The results of our study further expand perspectives of use of MPV in conditions associated with SLE with arthritis for monitoring treatment. So, more specifically designed prospective studies are needed to externally cross-validate our findings in a larger cohort of APS patients.

Authors' contributions:

Concept: SS; Data Collection and/or Processing: SS, AUU, SK; Interpretation: SS, TT, LA; Literature Review: SS, AUU, TT; Writer: SS, AUU, SK; Critical Review: SS. All authors read and approved the final manuscript.

Declaration of interest:

authors report no conflict of interest. 


\section{References}

1. Hopkinson ND, Doherty M, Powell RJ. Clinical features and race-specific incidence/prevalence rates of systemic lupus erythematosus in a geographically complete cohort of patients. Annals of the rheumatic diseases1994;53:675-680.

2. Osei-Bimpong A, Meek JH, Lewis SM. ESR or CRP? A comparison of their clinical utility. Hematology 2007;12:353-357.

3. Gaitonde S, Samols D, Kushner I. C-reactive protein and systemic lupus erythematosus. Arthritis and rheumatism 2008;59:1814-1820.

4. Pisetsky DS. Anti-DNA and autoantibodies. Current opinion in rheumatology 2000; 12:364-368.

5. Liang MH, Socher SA, Larson MG, Schur PH. Reliability and validity of six systems for the clinical assessment of disease activity in systemic lupus erythematosus. Arthritis and rheumatism 1989;32:1107-1118.

6. Petri M. Disease activity assessment in SLE: do we have the right instruments? Annals of the rheumatic diseases 2007;66:61-64.

7. Jackson SR, Carter JM. Platelet volume: laboratory measurement and clinical application. Blood reviews 1993;7:104-113.

8. Threatte GA. Usefulness of the mean platelet volume. Clinics in laboratory medicine 1993;13:937-950.

9. Yuksel O, Helvaci K, Basar O, Koklu S, Caner S, Helvaci N, Abayli E, Altiparmak E. An overlooked indicator of disease activity in ulcerative colitis: mean platelet volume. Platelets 2009;20:277-281.

10. Kisacik B, Tufan A, Kalyoncu U, Karadag O, Akdogan A, Ozturk MA, Kiraz S, Ertenli I, Calguneri M. Mean platelet volume (MPV) as an inflammatory marker in ankylosing spondylitis and rheumatoid arthritis. Joint, bone, spine: revue du rhumatisme 2008;75:291-294.

11. Endler G, Klimesch A, Sunder-Plassmann H, Schillinger M, Exner M, Mannhalter C, Jordanova N, Christ G, Thalhammer R, Huber K, Sunder-Plassmann R Mean platelet volume is an independent risk factor for myocardial infarction but not for coronary artery disease. British journal of hematology 2002;117:399-404.

12. Bath P, Algert C, Chapman N, Neal B. Association of mean platelet volume with risk of stroke among 3134 individuals with history of cerebrovascular disease. Stroke; a journal of cerebral circulation 2004;35:622626

13. Vila' LM AnG, McGwin Jr G, Bastian HM, Fessler BJ, Reveille JD. Systemic lupus erythematosus in a multiethnic cohort (LUMINA): XXIX. Elevation of erythrocyte sedimentation rate is associated with dis- ease activity and damage accrual. The Journal of rheumatology 2005;32:2150-2155.

14. de Carvalho JF HB, Szyper-Kravitz M, Shoenfeld Y. C-reactive protein and its implications in systemic lupus erythematosus. Acta Reumatol Port 2007;32:317322.

15. Gasparyan AY, Ayvazyan L, Mikhailidis DP, Kitas GD. Mean platelet volume: a link between thrombosis and inflammation? Current pharmaceutical design 2011;17:47-58.

16. Turner-Stokes L, Jones D, Patterson KG, ToddPokropek A, Isenberg DA, Goldstone AH. Measurement of haematological indices of chronic rheumatic disease with two newer generation automated systems, the H1 and H6000 (Technicon). Annals of the rheumatic diseases 1991;50:583-587.

17. Gasparyan AY, Sandoo A, Stavropoulos-Kalinoglou A, Kitas GD. Mean platelet volume in patients with rheumatoid arthritis: the effect of anti-TNF-alpha therapy. Rheumatology international 2010;30:11251129.

18. Makay B, Turkyilmaz Z, Unsal E. Mean platelet volume in children with familial Mediterranean fever. Clinical rheumatology 2009;28:975-978.

19. Sahin S, Senel S, Ataseven H, Yalcin I. Does mean platelet volume influence the attack or attack-free period in the patients with Familial Mediterranean fever? Platelets 2013;24:320-323.

20. Kapsoritakis AN, Koukourakis MI, Sfiridaki A, Potamianos SP, Kosmadaki MG, Koutroubakis IE, Kouroumalis EA. Mean platelet volume: a useful marker of inflammatory bowel disease activity. The American journal of gastroenterology 2001;96:776-781.

21. Lood C, Amisten S, Gullstrand B, Jonsen A, Allhorn M, Truedsson L, Sturfelt G, Erlinge D, Bengtsson AA. Platelet transcriptional profile and protein expression in patients with systemic lupus erythematosus: up- regulation of the type I interferon system is strongly associated with vascular disease. Blood 2010;116:1951-1957.

22. Nagahama M, Nomura S, Ozaki Y, Yoshimura C, Kagawa H, Fukuhara S. Platelet activation markers and soluble adhesion molecules in patients with systemic lupus erythematosus. Autoimmunity 2001;33:85-94.

23. Shanmugavelayudam SK, Rubenstein DA, Yin W. Effects of physiologically relevant dynamic shear stress on platelet complement activation. Platelets 2011;22:602610.

24. Larsson A, Egberg N, Lindahl TL. Platelet activation and binding of complement components to platelets induced by immune complexes. Platelets 1994;5:149-155. 